\title{
Path-Guided Wrinkling of Nanoscale Metal Films
}

\author{
Chuan Fei Guo, Vishal Naypar, Zhuwei Zhang, Yan Chen, Junjie Miao, \\ Rui Huang, * and Qian Liu**
}

Surface wrinkling instability is typically caused by compressive stresses in layered systems. In a bilayer system with a stiff surface layer on a drastically more compliant substrate, spontaneous wrinkle patterns emerge as a result of the wrinkling instability. The wrinkle patterns can be controlled to form ordered surface structures, offering an unconventional method for microfabrication. ${ }^{[1-22]}$ Several methods have been developed to control wrinkle patterns: by introducing bas-relief patterns into substrates, ${ }^{[1,9,10]}$ by locally modifying mechanical properties of substrates, ${ }^{[12,14,15]}$ by placing patterned PDMS mold on the surface, ${ }^{[13]}$ by applying anisotropic stress or strain,,$[2,5,11,17]$ by patterning the surface layer into ribbons and other shapes, ${ }^{[4]}$ and by tuning the adhesive properties of the interface. ${ }^{[18,22]}$ However, most of these techniques rely on unguided self-organization processes of wrinkle patterns, for which defects are inevitable. To generate high-quality wrinkle patterns with precise control of the shape and location, it is necessary to develop locally guided processes.

Here we present a new method for controlling wrinkle patterns in a gold/polystyrene (Au/PS) bilayer system. By locally modifying the mechanical properties of the gold film, we demonstrate high quality wrinkle patterns with various configurations. A similar method was reported by Huck et al.,[14] where they photochemically modified the surface of an elastomer before depositing a metal thin film. As a result, the effective mechanical properties of the composite surface layer (the metal film and the modified elastomer surface) were patterned and wrinkles were aligned accordingly. The key difference in the present method is that we directly modify the gold thin film using patterns with feature sizes much smaller than the intrinsic wrinkle wavelength $\left(\lambda_{i}\right)$ to precisely control the location and shape of wrinkles. We call such small features guiding paths (GPs), which can be lines, curves, dots, or other complex

Dr. C. F. Guo, Dr. Z. Zhang, Dr. J. Miao, Prof. Q. Liu National Center for Nanoscience and Technology No. 11 Beiyitiao, Zhongguancun Beijing 100190, China

E-mail: liuq@nanoctr.cn

V. Nayyar, Prof. R. Huang

Department of Aerospace Engineering

and Engineering Mechanics University of Texas

Austin TX 78712, USA

E-mail: ruihuang@mail.utexas.edu

Dr. Y. Chen

Institute of Mechanics

Chinese Academy of Sciences

No. 15 Beisihuanxi Road, Beijing 100190, China

DOI: 10.1002/adma.201200540 structures. In this study, we use laser direct writing (LDW) technique to make the GPs for guided wrinkling of nanoscale gold films.

The Au/PS bilayer has an intrinsic wrinkle wavelength $\lambda_{i}$ of $\sim 2.1 \mu \mathrm{m}$, which depends on the elastic moduli and the thicknesses of both layers (Supporting Information (SI), Section A). Typical feature size of the GPs by LDW is about $300 \mathrm{~nm}$, much smaller than $\lambda_{i}$. Figure $1 \mathrm{a}-\mathrm{b}$ illustrates the process of creating GPs, as well as guided wrinkle formation. Here the GPs serve as seeds for wrinkling, with the wrinkle crests forming exactly wherever laser writes. Figure $1 \mathrm{c}-\mathrm{k}$ shows atomic force microscopy (AFM) topographic images of a set of wrinkle patterns guided by LDW paths; the pitch of the GPs was designed to be around $2.1 \mu \mathrm{m}$ to match $\lambda_{i}$. These wrinkle patterns exhibit three unique features: i) they are highly ordered and defect-free; ii) the GPs define precisely the location and configuration of the wrinkles; iii) the guided wrinkle patterns can be lines, curves, dots, and more complex shapes. These wrinkle patterns demonstrate that various high-quality surface microstructures can be fabricated in a dry process that might be widely useful for high-throughput microfabrication (supplementary Figure S1). The path-guided wrinkling is especially suitable for fabricating large-area wavy surface structures (but not limited to, see other structures in supplementary Figure S2), which enable applications in phase gratings, microlenses (as demonstrated in the present study) and microfluidic channels.

To understand the underlying mechanism for the pathguided wrinkling, we show in Figure 2 a the wrinkle pattern as a result of one single GP by LDW. The wrinkle profile takes the shape of a highly damped wave, with the maximum amplitude at the location of GP. As the basic unit of the LDW-guided wrinkles, the wrinkle profile can be described mathematically as

$h(x)=A_{0} \cdot \cos \left(\frac{2 \pi}{\lambda_{i}} x\right) \cdot \exp \left(-\left|\frac{x}{l_{c}}\right|\right)$

where $A_{0}$ is the maximum height at the center of GP $(x=0)$, and $l_{c}$ is the effective damping length that can be determined experimentally (SI, Section B). The localized wrinkle profile is attributed to modification of the elastic modulus of the metal film as a result of LDW. The SEM image shows clearly lower densification and coarsened grains in the laser exposed area (SI, Figure S3), as a result of laser induced melting and resolidification. ${ }^{[23]}$ It has been reported that thermal annealing of $\mathrm{Au}$ films led to larger grains and lower modulus. ${ }^{[24]}$ Since laser writing typically leads to temperature rising similar to thermal annealing, the elastic modulus of the metal film is expected to be lower in the laser exposed area. To confirm this hypothesis, we performed numerical simulations of wrinkling based on a composite film model (SI, Section B and Figure S3). In 


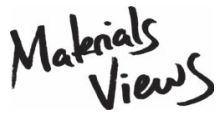

www.MaterialsViews.com
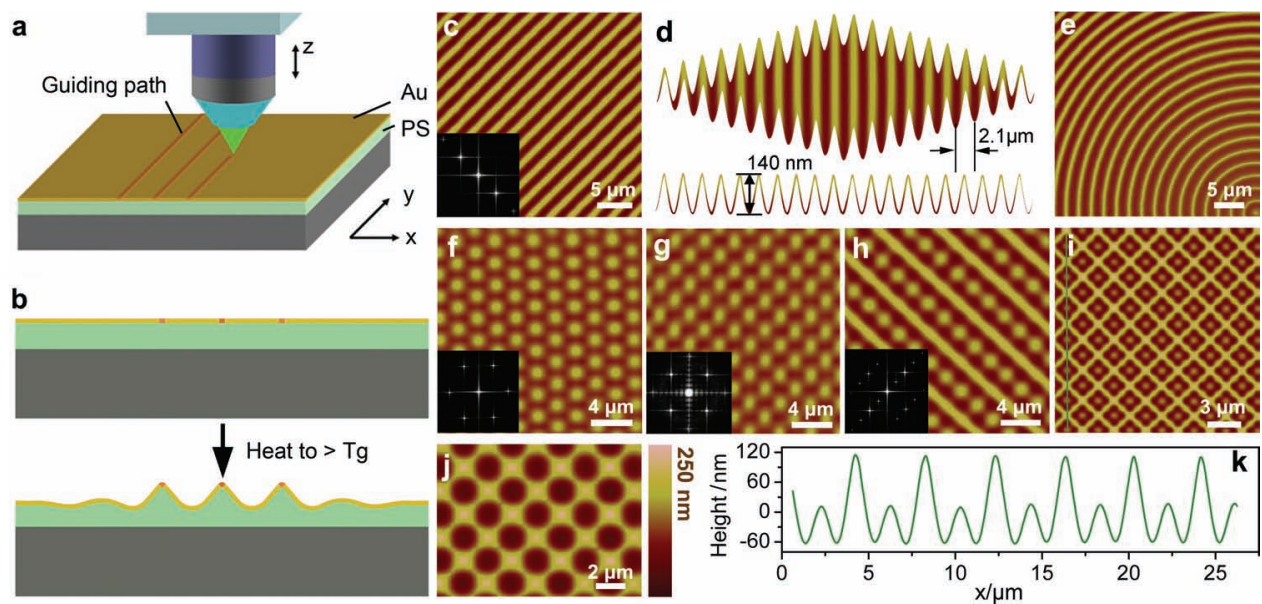

Figure 1. Fabrication of guided wrinkle patterns. (a) Schematic illustration of LDW for making GPs in a Au/PS bilayer. (b) Guided wrinkling along GPs upon heating. (c)-(j), AFM images of various surface microstructures: (c) parallel line wrinkles; (d) Tilt and side views of the wrinkles in (c), showing the highly uniform and periodic pattern. (e) Concentric circular wrinkles. (f), (g) Hexagonal and tetragonal arrays of wrinkle-dots, respectively. (h) A complex wrinkle pattern composed of dots and lines. Insets in (c) and $(f)$ - $(h)$ are the corresponding FFT spectra. (i) A wrinkle pattern composed of orthogonally aligned lines and dots. (j) An egg-crate structure. (k) Section analysis of the wrinkles along the green line in (i).

particular, a softening parameter $S$ is used to represent the effect of laser exposure, with the Young's modulus $E_{S}=S E_{m}(0<S<$ 1 ) for the metal film in the exposed area, while $E_{m}$ is the Young's modulus of the unexposed film. The softening parameter can be estimated by comparing the wrinkle wavelength in a large area exposed to laser with $\lambda_{i}$ in the unexposed area (SI, Section
C and Figure S4). By taking $S=0.4$, the simulated wrinkle profile agrees reasonably well with the experimental data, as shown in Figure 2a.

In contrast with the previous work by Huck et al., ${ }^{[14]}$ we note that the use of submicrometer feature size is essential for creating the localized wrinkle profile and hence precise control of the wrinkle patterns. When the feature size is much larger than $\lambda_{i}$, multiple wrinkle crests may appear within the laser exposed area (SI, Figure S5), similar to the wrinkle patterns obtained by Huck et al. ${ }^{[14]}$ Consequently, the wrinkle pattern cannot be fully controlled unless the feature size of the GP is sufficiently small. Moreover, it is noted that the pre-wrinkling stress distributions in the metal film depend on the feature size (SI, Figure S5). By using a small feature size, the stress outside the laser exposed areas is nearly unaffected while the stress in the laser exposed area becomes highly anisotropic. Therefore, the modification of the elastic property of the film by LDW also leads to a localized change in the stress state. By the conventional theory of wrinkling, ${ }^{, 25,26]}$ a homogeneous film with a lower Young's modulus would require a higher critical strain but a lower critical stress for the onset of wrinkling. However, for the composite film the critical condition for wrinkling in general cannot be predicted by the conventional theory. A similar phenomenon has been noticed recently for an elastic film on a compliant substrate with pre-existing interfacial delamination. ${ }^{[27]}$

By the mathematical description in Equation (1), we introduce a new concept of
Figure 2. Unit-wrinkle and interaction. (a) Surface profile of an unit-wrinkle, comparing the experimental result with numerical simulation based on a composite film model and the approximation by an exponentially damped wave function in Equation (1). Inset is the AFM image of the unit-wrinkle. (b) Calculated profiles of two parallel unit-wrinkles separated by a pitch distance of $3.6 \mu \mathrm{m}$ and the wrinkle profile obtained by superposition. (c) Comparison among the experimental, the numerical simulation, and the calculated wrinkle profiles with two parallel GPs (pitch $=3.6 \mu \mathrm{m}$ ). Inset is the corresponding AFM image. (d) AFM image and calculated topography of the wrinkle pattern with two perpendicular GPs. 
unit-wrinkle, which allows us to design more complex wrinkle patterns effectively. For example, two parallel GPs with a pitch distance $d$ would generate two unit-wrinkles interacting with each other. The interaction may be treated in a similar manner as interaction of waves so that the resulting wrinkle pattern is predicted by superposition of the two unit-wrinkles, e.g.,

$H(x)=k[h(x)+h(x-d)]$

where $h(x)$ and $h(x-d)$ are the height profiles of the unit-wrinkles as given by Equation (1), $k$ is a dimensionless parameter (SI, Figure S6). As shown in Figure 2b-c, the predicted wrinkle profile by Equation (2) agrees well with the measured profile with two parallel GPs. Moreover, numerical simulations based on the composite film model were also able to reproduce the wrinkle profiles with close agreement. The superposition method can be extended to more general two-dimensional cases so that the wrinkle profile composed of $n$ unit-wrinkles can be predicted using

$H(x, y)=k \sum_{i=1}^{n} h_{i}(x, y)$

where $h_{i}(x, y)$ represents the profile of one unit-wrinkle that depends on the location and orientation of the corresponding GP. Figure 2d shows an example with two perpendicular unitwrinkles, for which the wrinkle profile calculated by Equation (3) matches the experimental profile remarkably well. Therefore, by using Equation (3), the surface profile of various wrinkle patterns can be designed and then realized by the processes of LDW and path-guided wrinkling. As an unconventional method to microfabrication, the LDW path-guided wrinkling has uniquely versatile controllability in four aspects: a) selected area patterning, b) accurate alignment, c) tunable wavelength, and d) height control.

With the localized profile of each unit-wrinkle, wrinkle patterns can be obtained with the maximum amplitude in the exposed areas only (hence selected area patterning). This behavior also provides us the opportunity to re-fabricate wrinkle patterns in featureless areas. By rewriting and reheating, new wrinkle patterns can be generated on top of existing patterns (SI, Figure S7), similar to the alignment process in photolithography.

Wrinkles formed by previous methods typically have an intrinsic wavelength, $\lambda_{i}$. Guided by LDW paths, the wrinkle wavelength can be tailored by the pitch of GPs. For the Au/PS bilayer system with an intrinsic wrinkle wavelength of $2.1 \mu \mathrm{m}$, the LDW-guided wrinkle wavelengths ranging from $\sim 0.6$ to $\sim 2.8 \mu \mathrm{m}$ can be obtained (SI, Figure S8). The range of the tunable wrinkle wavelength depends on the intrinsic wrinkle wavelength of the bilayer system. By using GPs with smaller feature sizes (e.g., by using a scanning near-field optical microscope) and a bilayer system with a submicron $\lambda_{i}$, guided wrinkle patterns with wavelengths less than $600 \mathrm{~nm}$ may be achieved. In particular, the tunability of the wrinkle wavelength is useful for making surface structures with different periods such as Fresnel lenses.

In addition, the wrinkle height can be controlled by adjusting the laser exposure dose in selected areas. With the capability of selected-area patterning, we could locally modify

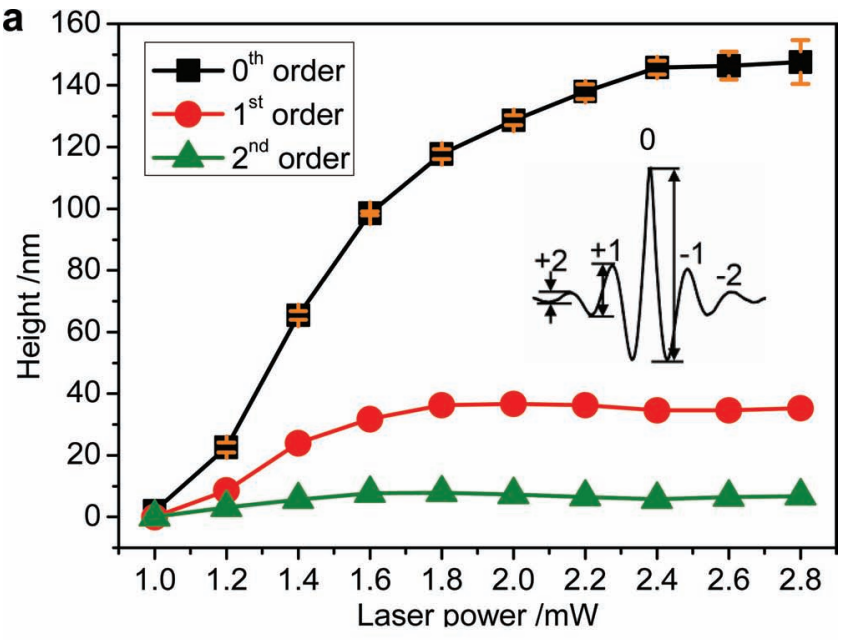

b

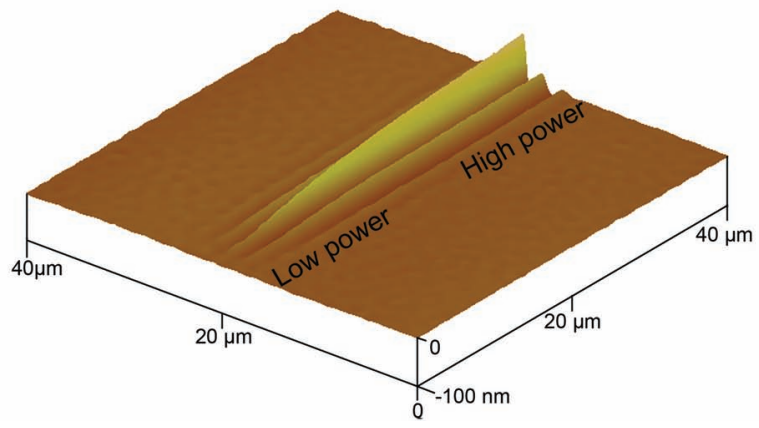

Figure 3. Height control of wrinkles. (a) Wrinkle height versus laser power, showing that wrinkle height increases with increasing laser power. The inset defines the wrinkle amplitudes $\left(0^{\text {th }}, 1^{\text {st }}\right.$, and $2^{\text {nd }}$ orders $)$. (b) Tilt view of an AFM image showing the wrinkle pattern with the height varying continuously along the guiding path due to varying laser power. The variation of the wrinkle height with the laser power is shown in SI, Figure S10.

the elastic modulus of the metallic film and thus control the wrinkle height selectively. As shown in Figure 3a, the height of the unit-wrinkle increases with the laser power within a certain power range $(1.0-2.8 \mathrm{~mW})$. This can be understood as a result of decreasing elastic modulus in the laser exposed area due to increasing laser power, as shown by numerical simulations (SI, Figure S9). Interestingly, by varying the laser power along one straight GP, the height of the wrinkle can be controlled continuously along the path (e.g., from 0 to $148 \mathrm{~nm}$ in Figure $3 \mathrm{~b}$ and SI, Figure S10). Furthermore, we note that the unit-wrinkle can have a much larger height-to-wavelength aspect ratio than typical wrinkle patterns $(A / \lambda \sim 0.1)$. By heating the bilayer to a higher temperature and holding for a longer time, a higher wrinkle amplitude can be achieved while the wrinkle wavelength does not change significantly, as predicted by the theory of viscoelastic wrinkling. ${ }^{[25]}$ A unit-wrinkle with a height of $586 \mathrm{~nm}$ and a width of $\sim 1700 \mathrm{~nm}$ was demonstrated (SI, Figure S11), corresponding to an aspect ratio of 0.34 . Wrinkles with high aspect ratios have been reported elsewhere, ${ }^{[21]}$ but the wrinkle patterns were not well controlled previously. 

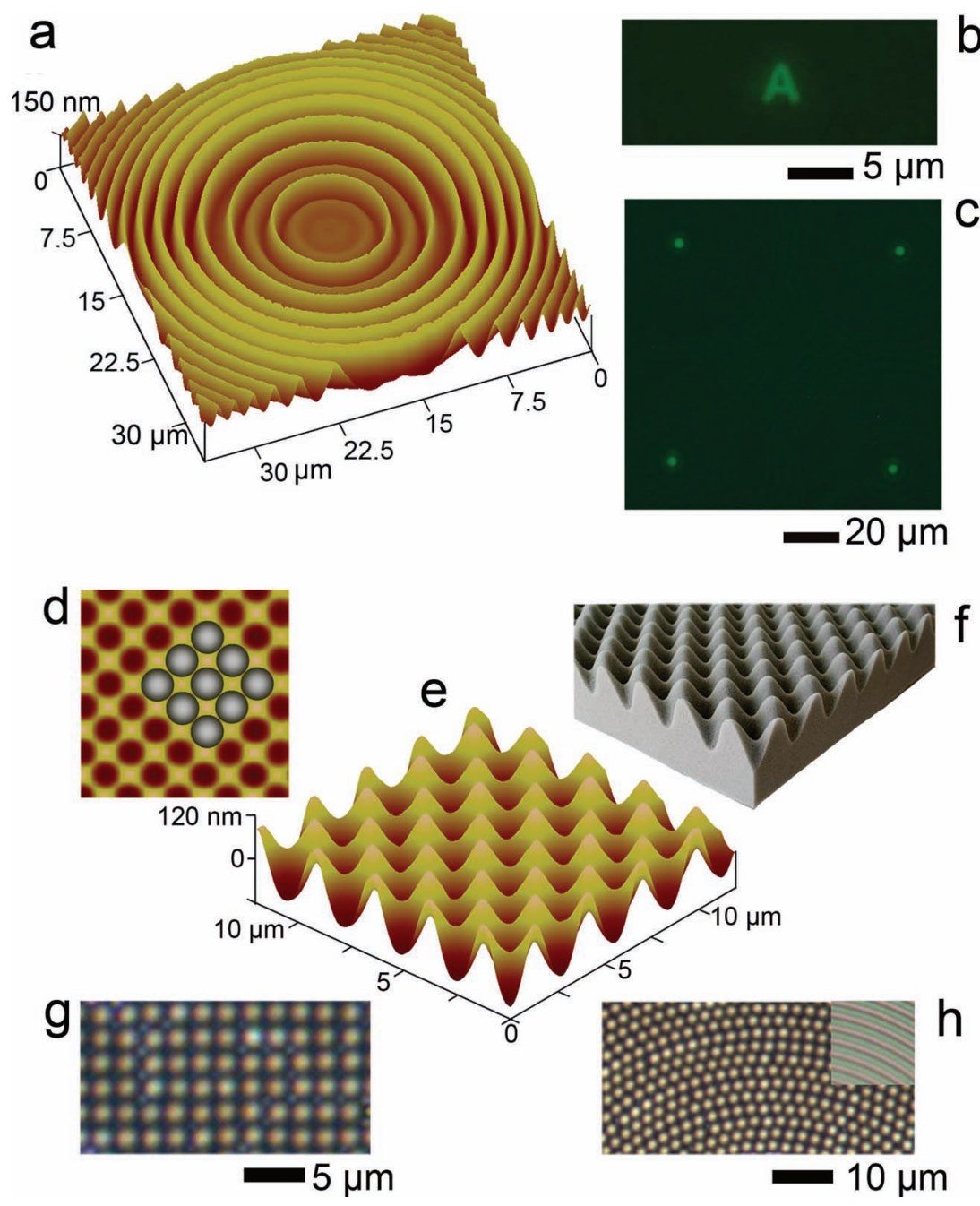

Figure 4. Fabrication of wrinkle devices. (a) AFM image of a Fresnel lens made up of concentric wrinkles. (b) Imaging effect (showing a letter "A") of the Fresnel lens. (c) Focusing effect of a $2 \times$ 2 lens array. (d) An egg-crate structure used as template for aligning microspheres. (e) Tilt view of the egg-crate structure. ( $f$ ) A real egg-crate. (g) Tetragonal PS microsphere lattice directed by an egg-crate wrinkle structure. (h) PS microspheres aligned in a concentric pattern. The inset shows the optical image of the template made up of concentric circular wrinkles.

The high-quality and controllable wrinkle patterns could have many applications. Figure 4a-c demonstrates focusing and imaging effects of a Fresnel lens (and a lens array) composed of non-periodic wrinkles. Without the wavelength control, such devices are unachievable. Figure 4d-h show periodic wrinkle patterns as the templates for aligning colloidal microspheres into a tetragonal lattice and concentric rings. The microspheres with a diameter matching the wrinkle wavelength exactly fall on the troughs (SI, Figure S12). Moreover, a two dimensional (2D) sinusoidal grating beam splitter made up of wrinkles was demonstrated (SI, Figure S13). We believe that surface microstructures made by path-guided wrinkling can be applied to many fields such as diffractive optical elements, MEMS, solar cells, wave absorbers and biomimetic tissues. Furthermore, the pathguided wrinkling behavior has also been observed in a Sn/PS bilayer system (SI, Figure S14), which suggests that the method can be extended to other material systems. from the author.
In summary, we present a new method to obtain high quality and highly controllable wrinkle patterns. By introducing subwavelength GPs, we can accurately design and fabricate desired surface microstructures by path-guided wrinkling, including periodic and non-periodic patterns. The underlying mechanism for the guided wrinkling is primarily attributed to the effect of laser direct writing that lowers the elastic modulus in the exposed areas of the metal film. Numerical simulations based on a composite film model supported the hypothesis. A new concept, unit-wrinkle, is suggested as a useful tool for designing complex wrinkle patterns by the method of superposition. A variety of wrinkle patterns are demonstrated. As an unconventional method to microfabrication, LDW path-guided wrinkling has uniquely versatile controllability, especially for fabrication of wavy surface microstructures. We expect to create high-quality wrinkles for applications in many devices which are unavailable or too costly by conventional techniques.

\section{Experimental Section}

Wrinkle pattern generation: A toluene solution of PS (with a molecular weight of 100000 ) was firstly spin-coated on a glass substrate and then annealed at $60^{\circ} \mathrm{C}$ for $12 \mathrm{~h}$ to remove residual solvent and relieve stress, forming a PS film (thickness 300-350 nm). The PS film was then covered by sputtering a $\mathrm{Au}$ film (thickness 5-7 $\mathrm{nm}$ ). A laser direct writer (NanoLDW-I, laser wavelength of $532 \mathrm{~nm}$, laser spot size of $\sim 300 \mathrm{~nm}$, pulse width of $200 \mathrm{~ns}$, and laser power of 1-5 $\mathrm{mW}$ ) was used to make GPs, e.g., lines, curves, and dots, in the Au film. After laser writing, the Au/PS bilayer was heated to $\sim 120{ }^{\circ} \mathrm{C}$ (slightly above the glass transition temperature of PS, $T_{g} \sim 105^{\circ} \mathrm{C}$ ) and held for a duration (10 min to $2 \mathrm{~h})$ to generate wrinkle patterns.

Characterization: Observation of the wrinkle structures was performed by optical microscopy (Leica DM 2500); surface profiles were measured by AFM (Veeco D3100); and film morphology was observed by scanning electron microscopy (SEM, Hitachi S4800). Focusing and imaging experiments of Fresnel lenses were performed in a Leica microscope using a $540 \mathrm{~nm}$ light source by using the same method as in Ref. [3].

Self-assembly of PS microspheres: $50 \mu \mathrm{L}$ aqueous suspension of PS microspheres ( $2 \mathrm{wt} \%$, with a slight anionic charge from surface sulfate groups and a diameter of $2 \mu \mathrm{m}$ ) from Alfa Aesar was first added to $1.0 \mathrm{~mL}$ isometric ethanol. A drop of such suspension $(5 \mu \mathrm{L})$ was then dripped onto the wrinkled surface, which is hydrophilic, and finally the PS microspheres were self-assembled following the wrinkle patterns.

\section{Supporting Information}

Supporting Information is available from the Wiley Online Library or 


\section{Acknowledgements}

The experimental part of this work was supported by the funds from NSFC (10974037), NBRPC (2010CB934102), International S\&T Cooperation Program (2010DFA51970) and Eu-FP7 (No. 247644). The modeling and simulations were supported by the US National Science Foundation (Grants No. 0926851). We thank Dr. Zhang Jianming for helpful discussions.

Received: February 7, 2012

Revised: March 20, 2012

Published online: May 2, 2012

[1] N. Bowden, S. Brittain, A. G. Evans, J. W. Hutchinson, G. W. Whitesides, Nature 1998, 393, 146.

[2] C. Harrison, C. M. Stafford, W. Zhang, A. Karim, Appl. Phys. Lett. 2004, 85, 4016.

[3] E. P. Chan, A. J. Crosby, Adv. Mater. 2006, 18, 3238.

[4] Y. Sun, W. M. Choi, H. Jiang, Y. Huang, J. A. Rogers, Nature Nanotechnol. 2006, 1, 201.

[5] K. Efimenko, M. Rackaitis, E. Manias, A. Vaziri, L. Mahadevan, J. Genzer, Nat. Mater. 2005, 4, 293.

[6] C. M. Stafford, C. Harrison, K. L. Beers, A. Karim, E. J. Amis, M. R. VanLandingham, H.-C. Kim, W. Volksen, R. D. Miller, E. E. Simonyi, Nat. Mater. 2004, 3, 545.

[7] C. M. Stafford, B. D. Vogt, C. Harrison, D. Julthongpiput, R. Huang, Macromolecules 2006, 39, 5095
[8] Y. Chung, T. Q. Chastek, M. J. Fasolka, H. W. Ro, C. M. Stafford, ACS Nano 2009, 3, 844.

[9] S. J. Kwon, P. J. Yoo, H. H. Lee, Appl. Phys. Lett. 2004, 84, 4487.

[10] N. Bowden, W. T. S. Huck, K. Paul, G. W. Whitesides, Appl. Phys. Lett. 1999, 75, 2557.

[11] T. Ohzono, M. Shimomura, Phys. Rev. B 2004, 69, 132202.

[12] T. Ohzono, S. I. Matsushita, M. Shimomura, Soft Matter 2005, 1, 227.

[13] P. J. Yoo, K. Y. Suh, S. Y. Park, H. H. Lee, Adv. Mater. 2002, 14, 1383.

[14] W. T. S. Huck, N. Bowden, P. Onck, T. Pardoen, J. W. Hutchinson, G. M. Whitesides, Langmuir 2000, 16, 3497.

[15] M. Muller-Wiegand, G. Georgiev, E. Oesterschulze, T. Fuhrmann, J. Salbeck, Appl. Phys. Lett. 2002, 81, 4940.

[16] C. Jiang, S. Singamaneni, E. Merrick, V. V. Tsukruk, Nano Lett. 2006, 6, 2254.

[17] P.-C. Lin, S. Yang, Appl. Phys. Lett. 2007, 90, 241903.

[18] H. Vandeparre, J. Léopoldès, C. Poulard, S. Desprez, G. Derue, C. Gay, P. Damman, Phys. Rev. Lett. 2007, 99, 188302.

[19] H. Vandeparre, P. Damman, Phys. Rev. Lett. 2008, 101, 124301.

[20] J. Y. Chung, A. J. Nolte, C. M. Stafford, Adv. Mater. 2009, 21, 1358.

[21] S. F. Ahmed, G. H. Rho, K. R. Lee, A. Vaziri, M.-W. Moon, Soft Matt. 2010, 6, 5709.

[22] G. M.- Garnier, A. B. Croll, C. S. Davis, A. J. Crosby, Soft Matt. 2010, 6, 5789.

[23] C. F. Guo, Z. Zhang, S. Cao, Q. Liu, Opt. Lett. 2009, 18, 2820.

[24] R. D. Emery, G. L. Povirk, Acta Mater. 2003, 51, 2067.

[25] R. Huang, J. Mech. Phys. Solids 2005, 53, 63.

[26] Z. Y. Huang, W. Hong, Z. Suo, J. Mech. Phys. Solids 2005, 53, 2101.

[27] H. Mei, C. M. Landis, R. Huang, Mech. Mater. 2011, 43, 627. 